\title{
Household Appliance Product Appearance Design with Feedback of User Experience
}

\author{
Shan $\mathrm{Du}^{1}$ \\ ${ }^{1}$. Zhengzhou University of Aeronautics, Zhengzhou 450015, China \\ E-mail:23622705@qq.com
}

\begin{abstract}
Household appliance product design is a big trend in current industrial design circle. Reverse engineering is one of the most common CAID means in modern industrial design. In order to support reusing product appearance design knowledge, this paper proposes the model used for resolving innovative product appearance case. According to the three stages of aesthetics impression, semantic judgment and symbol contact experienced by users to cognize product, this paper divides the product into three kinds of appearance innovative types of formal aesthetics, usage mode and cutural fashion By utilizing taxonomy principle and appearance design innovative process, this paper sets up three kinds of appearance innovative types corresponding to design knowledge frame model used for extracting and reusing appearance innovative design knowedge. Such model is applied to setting up product innovative case ilorary, helping designer search and apply previous successful cases and realizing user-centered product development. Through the use of such database by designer, this paper shows its effectiveness.
\end{abstract}

Keywords: Appliance product:-Reverse engineeving; Ergonomics; User experience with feedback; Systematic integration.

\section{Introduction}

Reverse engineering technology is a newly-developing subject which develops rapids in recent years, also called inverse-seeking engineering. It includes shape reverse engineering, technology reverse engineering and material reverse engineering. However, current reverse engineering technology researches mostly focus on geometric model revere engineering based on part and sample piece, that is to produce corresponding CAD model from the existed physical model or part and then improve design and fabrication. At present, market Competition is fiercer, product technological content is improved constantly and abrication cycle is shortened constantly, reverse engineering technology has been emphasized more and more.

Reverse engineering mainly depends on computer technology and network technology of high-degree integration, visualization and openness to construct product and realize paperless, highly accurate and systematic operation platform from concept design, product design, engineering analysis, technological manufacturing, application engineering and market service in the whole process. Nowadays, we still need fabricate sample for physical test, argument and evaluation, but it has not been an important means and will be cancelled finally. For this thinking method, thinking is prior to real object and real object is used to disprove reverse logical form of thinking. International manufacturing circle call it reverse engineering. The purpose of carrying out reverse engineering is to realize the parallel engineering of product design better, make product design and related process synchronized, makes it optimized and improve one-time successful rate of product design so as to shorten cycle, reduce cost, reduce risk, improve quality and strengthen corporate competitiveness. 


\section{Reverse Engineering}

Traditionally, the development of industrial product follows development process with rigid sequence, which is development procedure of forward product. Designer draws sketch and design sketch according to the stated planning and design in product planning stage, makes handmade model or construct CAD geometrical model according to design sketch and conducts NC or RP processing procedure at last. Such engineering development is called forward engineering. The process of forward engineering is shown in Diagram 1 and 2.

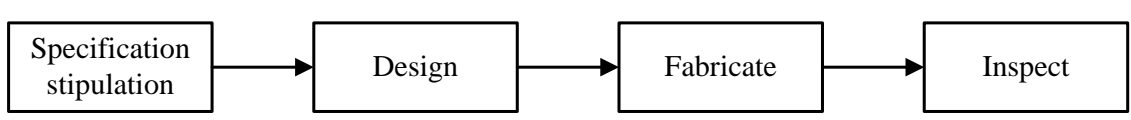

\section{Diagram 1. Forward Engineering Development Process of Component}

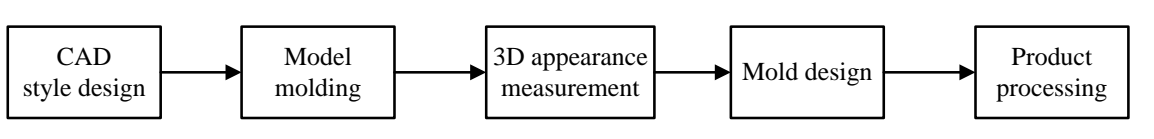

Diagram 2. Product Style Design Processing Flow in Forward Engineering

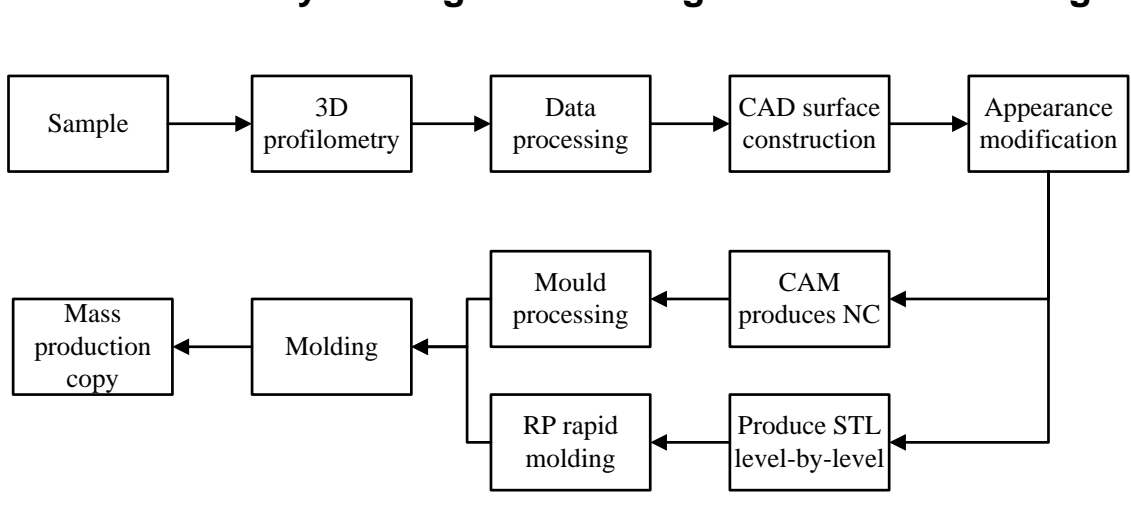

Diagram 3. Reverse Engineering Flow Chart

Reverse engineering usually execute copy and development work of model. Reverse engineering based on prototype or real object reduces development cycle of product greatly owing to the rapid and convenient development method, thus it has wide technological demand in design and manufacturing field. Especially for parts without original geometrical mformation, reverse engineering is the sole method to complete accurate geometrical model of parts, so it becomes technological hot topic in mould design and manufacturing. Meanwhile, reverse engineering is able to provide main appearance feature of original product, modify its geometrical appearance and realize rapid modification of product, so it can meet the requirement of seriation and diversification. Traditional copy method is to use 3D carving machine or profiling machine to make $1: 1$ isometric mould and then carry out volume production. Such copy method cannot establish picture file of workpiece dimension nor do any appearance modification, so it has been replaced by digital reverse engineering system. At present, so-called reverse engineering is to utilize $3 \mathrm{D}$ digital measuring instrument to measure point data or contour line on surface of current workpiece rapidly, create surface, split mold, process and manufacture the required mold; or transmit STL model file produced by CAD system to rapid prototyping machine, make sample model until up to the satisfying effect. In many reverse engineering application cases, it doesn't mean to copy original product totally, but to master original design idea and establish a similar design model after adjustment, so the covered meaning of reverse engineering is not merely re- 
manufacturing, it also includes re-design, as is shown in Diagram 3. Therefore, reverse engineering can be reduced to: functionally-oriented, descriptive mode, system as-is and non-legacy system.

\section{Realization Process of Reverse Engineering}

Reverse engineering is usually used for the process of copy. That is to carry out 3D digital processing for real object. Digital means includes traditional surveying and various advance measurements. Take acquired SD discrete data as initial material, borrow special surface processing software and $\mathrm{CAD} / \mathrm{CAM}$ system to construct $\mathrm{CAD}$ model of real object, input NC processing command or use STL file to drive rapid prototyping machine to manufacture product or prototype, the technological process is shown in Diagram 1. Of them, full line part is technological process of reverse engineering discussed in this paper and dotted portion is another technological route of reverse engineering. 3D date, measurement, data processing and 3D reconstruction are three key technologies of reverse engineering. (1) Geometrical measurement: gain 3D shape of product through proper measuring methods; (2) data processing: process the acquired 3D data o eonform to follow-up operation; (3) CAD modeling: set up a complete $\mathrm{CAD}$ model to describe total related information of product.

\subsection{D Data Collection}

In reverse engineering, acquiring 3D geometrical data of real object correctly, rapidly and comprehensively, which is 3D discrete digital processing for 3D geometrical surface of object, is basis of realizing reverse êngineering. Data collection means using certain measuring method and equipment to measure geomerrical coordinate of several group points on surface of real object. There ntay be various kinds of data collection. In surface digital technology, data collection methods can be divided into contact type and noncontact type according to measuring methods. Traditional method is contact type with coordinate measuring machine (CMM) as representative, which is also the common method in real engineering. The precision is relatively accurate, but it easily damages measuring head and scratches the sufface of tested parts.

\subsection{Data Processing}

Data processing is a ke step of reverse engineering and the result will affect the quality of later model reconstruction directly. The process includes the following work: (1) data preprocessing (2) data segmentation; (3) data smoothing; (4) data optimization.

In Pro/E program, select main menu command [File]/[New] to create a new entity part, then select main menu command [Application]/[Scan-tools], assign data density mode (low density mode), set up coordinate system, read data and generate curve. If original measured data has great error, we must use remove scan point to remove the point with big derivation. We can also use create scan curve, join scan curve and separate san curve nethods to process curve directly and gain satisfied curve. After generating scan curve, we can create smooth curve, adjust and smooth curve with number of points' method.

\subsection{CAD Modeling}

In reverse engineering, the reconstruction of 3D CAD model is to utilize scattered points on surface of product to construct a similar model to approach product prototype through interpolation or fitting. The reconstruction of 3D CAD model is the most key and most complicated link in the whole reverse engineering and the basis of follow-up product processing, rapid prototyping, engineering analysis and product redesign. 3D CAD modeling of product means the process of producing corresponding CAD model from an existed physical model or real part, including object discrete measuring point gridding, 
feature extraction, surface fragmentation and surface generation, etc. which is the most key and most complicated link in the whole RE process and also provides mathematical model support for follow-up engineering analysis, innovative design and processing and manufacturing. The content involves multiple interdisciplines and engineering field such as computer, photoshop, graphics, computation geometry, measurement and numerical control processing, etc. It is hot topic and difficult problem widely concerned by foreign and domestic academic circles, especially in CAD/CAM field.

In real product, it's usual to have one surface of composition. Product surface is usually made of various mixed surfaces. The type of surface is different, so the general procedure of $\mathrm{CAD}$ model reconstruction is: segment point cloud data according to geometrical feature firstly, fit each surface piece respectively and "sew up" various surfaces into a whole through transition, intersection, cutting and rounding, etc. that is reconstruct $\mathrm{CAD}$ model. Pro/E has many powerful modules, such as part modeling module, engineering drawing module, numerical control processing module, etc. We use part modeling module to inverse seek modeling here and create 3DCADModel under part module. After creating 3DCADModel, we can use View/Advance $\mathrm{d} \rightarrow$ Photorender to make high-quality renderings further.

\subsection{Numerical Control Processing}

Import the created 3DCADModel to Manufacturing module of Pro/E, set up new Pro/NC file (create new Pro/NC mockup) and select NC module option button under New Menu. Select Manufacture Model option in Menu Manger select Reference Model and Open Modeling Model in Assembly. Select manufacturing setting, set up various parameters, including setting of machine tool, cutter fixture, reference and retract, etc. then conduct processing setting, select NO sequence in processing, select profile option in processing and then conduct sequence setting, including choice of cutter and curve, and parameter setting, etc. and process outer contour line of cam through contour processing method.

After confirming processing process i correct, we can output program. Select CL data option in Menu Manger select output-track-file, and select CL file and MCD file option, then output and generation of NCprocessing program is completed. Until now, after the above inverse seeking modeling and generation of NC processing program, set numerical control processing codes generated by Pro/E manually, send them to electric cremation line cutting machine or processing center for processing, then inverse seeking design and manufacturing of moulc part is completed.

\section{User's Cognition Research}

\subsection{Desigh Scheme Establishment}

At the stage of design scheme establishment, designers need refer to universal available interface of cognitive load of users to design matrix, establish interactive design scheme directing at cognitive load of users and make preparation for development of experimental prototype system.

Detailed interactive design scheme establishment depends on specified interactive task, so designers still need refer to related digital reading interface interactive design criteria. At this stage, interactive design mainly appears in the form of design document, and design document should include detailed information architecture method, interface pattern, interface element, task process and interface sequence. 


\subsection{Prototype System Architecture}

At the stage of system realization, after determining interactive design scheme, designers should refer to interactive design document to design and realize specific interface content. Combing with general available matrix and design scheme of digital reading can help to design interface information easily understood by users with different cognitive levels.

In order to realize complete interaction process and make users experience real visual effect, we can apply FLASH, etc. interface design tool to realize it.

\subsection{Feedback Mode of User Experience}

Internal process in Diagram 4 is the basic sequence of system operation perceived by users according to self-knowledge and the current state of system and external process is real operation sequence of system. Is the process perceived by users is in line with real process of system, then operation will be done normally according to sequence, until goal completion; once the process perceived by users disagrees with the real process of system, it will obstruct the smooth running of interaction. Now, designers should analyze optimal operation sequence according to the perception of operation process by users and adjust original sequence of interaction process. For example, plovide operation process demonstration for new user or provide operation schedule reminder bar to make user understand operation history and current schedule clear y.

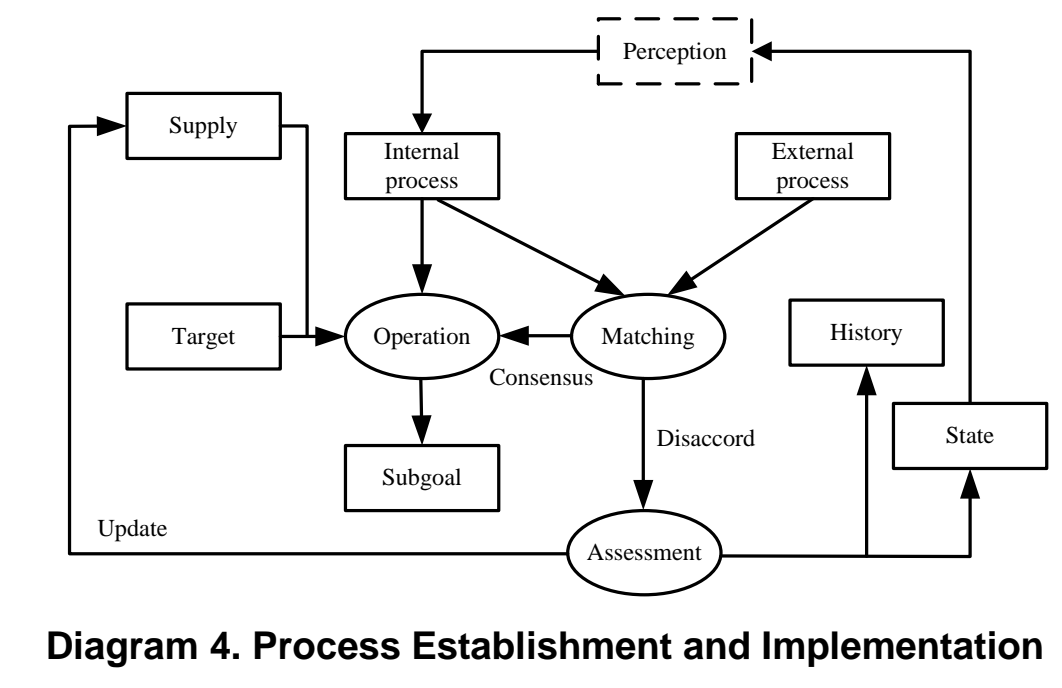

Supply identification and acquisition: whether to acquire correct supply relation or not concerns the success of the whole interaction task. In the current interactive process of system, aser identifies executable operation according to self-knowledge, current situation and system state, realize the transition of current goal to next goal through a series of operations and confirm acquisition of supply by combining goal assessment. Detailed description is as shown in Diagram 5. 


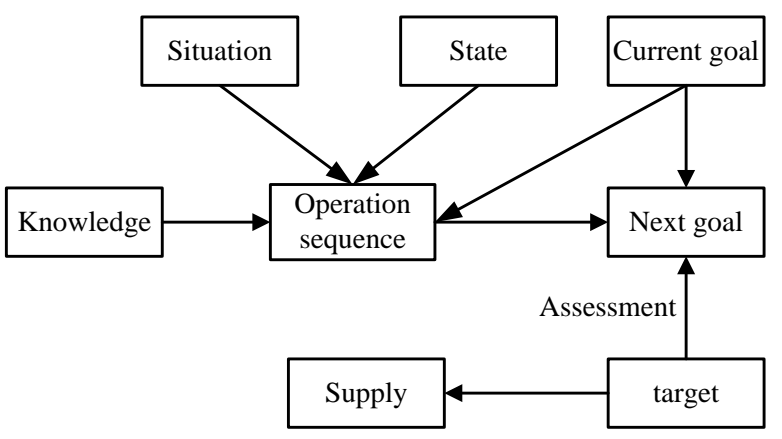

\section{Diagram 5. Supply Identification and Acquisition}

The above information structure and interactive strategy are sufficient for simple human-computer interaction toward digital reading. It can provide a coherent core for interaction modeling of digital reading interface and guide the establishment of design method.

\subsection{Cognitive Load Assessment}

Cognitive load level has direct relation with supply identification/and acquisition. Related researches find that operation performance is not directly with cognitive load level and some high-performance experimental scheme usually cannot get higher subjective satisfaction of users, this is because users produce higher cognitive load when interacting with system. Therefore, it's necessary to assess cognitive load of users. Cognitive load of users in human-computer interact on cannot be measured directly at present, which can only be evaluated through indirect related indexes.

The assessment results are shown as in Diagram, 6 to 8. They represent cognitive load indexes, satisfactions and operation perfornances of registered task of two prototype systems operated by users. According to statistic result, cognitive load, satisfaction and performance of three tasks operated by users with prototype system is superior to various indexes by using reference system.

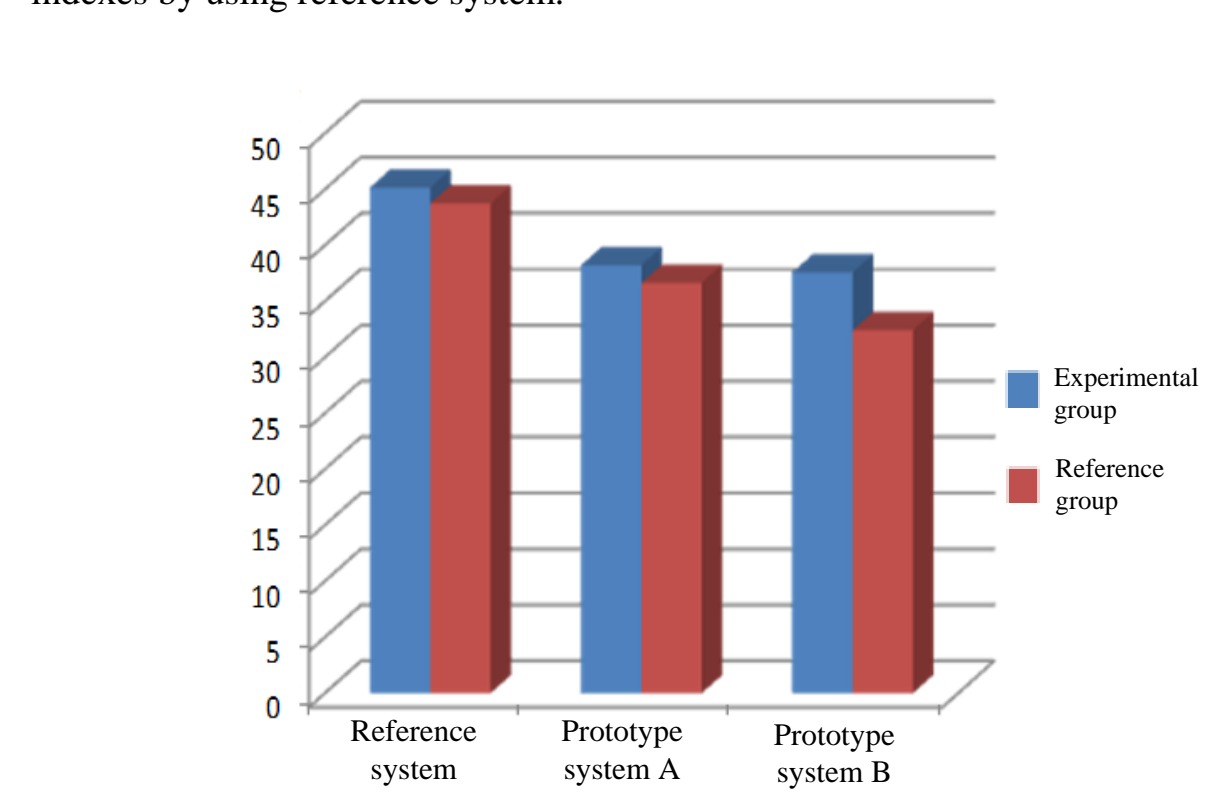

Diagram 6. Average Cognitive Load Level Contrasts 


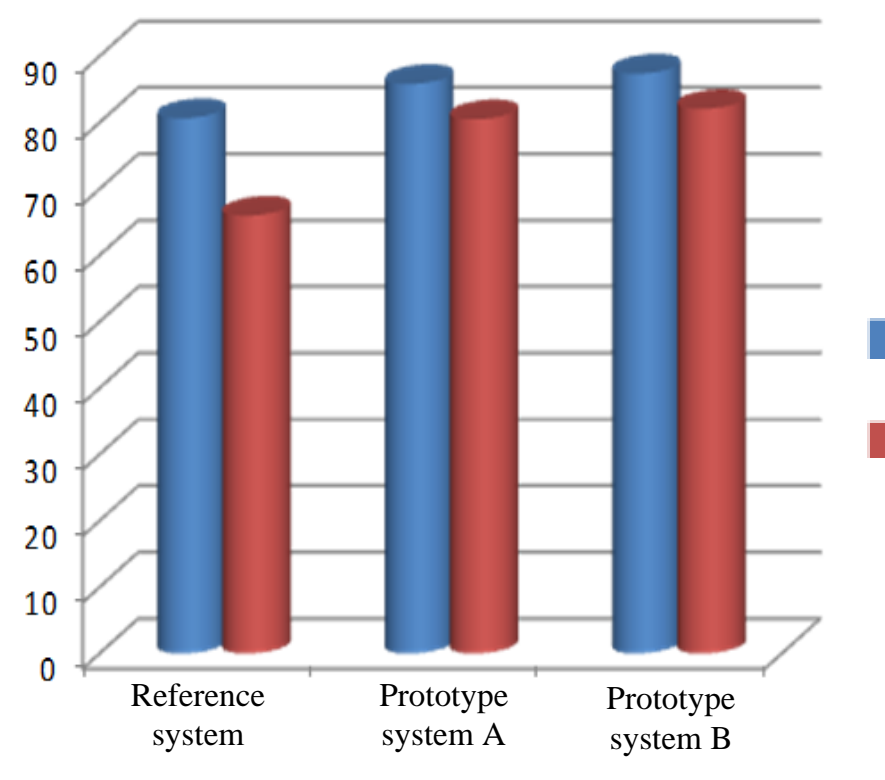

Reference group

Experimental group

Diagram 7. Average System Satisfaction Contrasts

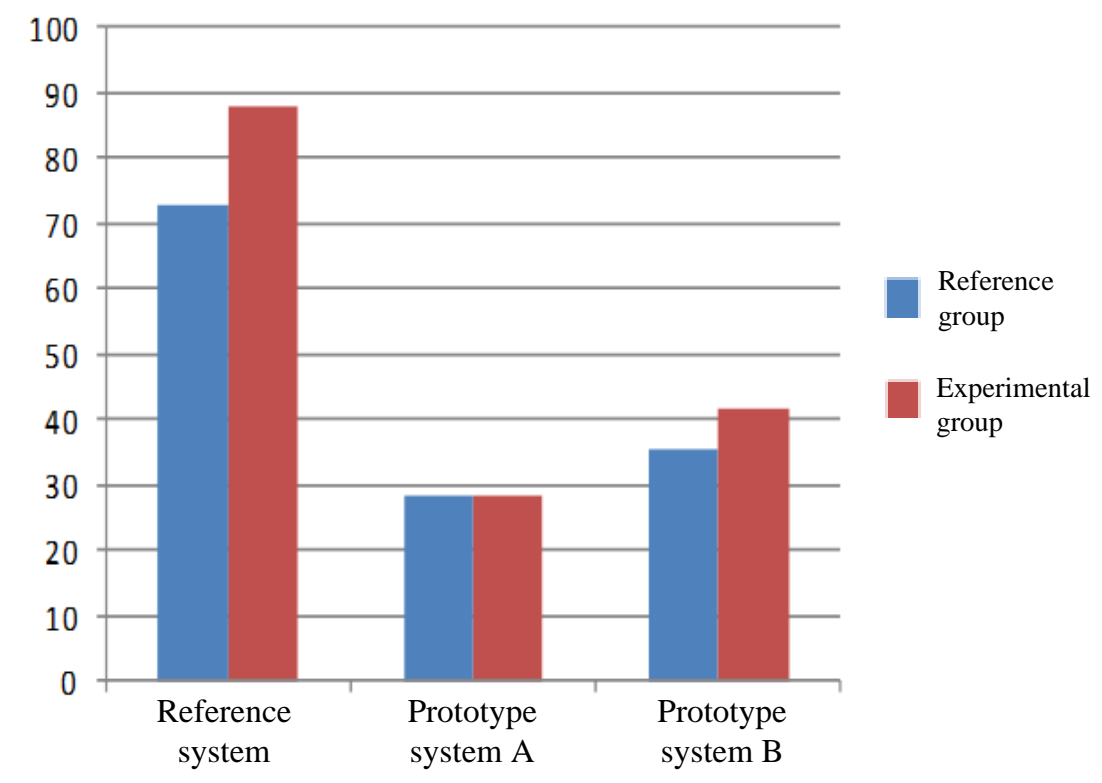

Diagram 8. Average Completion Time Contrasts

Throgh applying the acquired date to independent sample $\mathrm{T}$ test, we find that there are significant differences between the satisfaction of reference system by experimental group users and reference group users and there are no significant differences between the satisfaction of experimental prototype system A and experimental prototype system B. It shows that reference system doesn't take full consideration of interactive experiment among aged users, new users and low degree of education users, while experimental prototype system takes full consideration of special user group as well as without affecting operation experience of mainstream user group. Therefore, interactive design method toward digital reading interface discussed in this paper is effective to some extent. 


\section{Conclusion}

Reverse engineering, especially $3 \mathrm{D}$ model reconstruction is still a strongly professional job. Except understanding product characteristic, manufacturing method, mastering CAD software and reverse molding software, we should be familiar with the upstream measuring device and measuring process to understand data structure and the requirement of reconstructing model in follow-up processing. Therefore, the quality of model reconstruction is still affected by experience and level of operator. How to improve intelligence of software and reduce the dependency on designers is the direction of development in the future.

\section{Acknowledgement}

The research is supported by Research on the application of green logistics packaging, design in Zhengzhou airport economic comprehensive experimentation area No. 142400410803).

\section{References}

[1] Lv Z. Wearable smartphone: Wearable hybrid framework fon hand and foot gesture interaction on smartphone $[\mathrm{C}] / /$ Computer Vision Workshops (ICCVW) (2013) IEEE International Conference on. IEEE, 2013: 436-443.

[2] Lin Y, Yang J, Lv Z. A Self-Assessment Stereo Capture Model Applicable to the Internet of Things[J]. Sensors, (2015), 15(8): 20925-20944.

[3] Yang J, He S, Lin Y. Multimedia cloud transmission and storage system based on internet of things[J]. Multimedia Tools and Applications, (2015)\& 1- 6 .

[4] Lv Z, Yin T, Han Y. WebVR — web virtual reality engine based on P2P network[J]. Journal of Networks, (2011), 6(7): 990-998.

[5] Yang J, He S, Lin Y. Multimedia cloud transmission and storage system based on internet of things[J]. Multimedia Tools and Applications, (2015).

[6] Guo C, Liu X, Jin M. The research on optimization of auto supply chain network robust model under macroeconomic fluctuations[J]. Chaos, Solitons \& Fractals, (2015).

[7] $\mathrm{Li} \mathrm{X}, \mathrm{Lv} \mathrm{Z}, \mathrm{Hu} \nrightarrow \mathrm{XEarth}$ : A $3 \mathrm{D}$ GIS Platform for managing massive city information[C]//Computational Intelligence and Virtual Environments for Measurement Systems and Applications (CIVENSA), 2015 IEEE International Conference on. IEEE, (2015): 1-6.

[8] Yang J, Chen B, Zhou J. A Low-Power and Portable Biomedical Device for Respiratory Monitoring with a Stable Power Source[J]. Sensors, (2015), 15(8): 19618-19632.

[9] Guanqun Bao, Liang Mi, Yishuang Geng, Kaveh Pahlavan, A computer vision based speed estimation technique for localiz ing the wireless capsule endoscope inside small intestine, 36th Annual International Conference of the IEEE Engineering in Medicine and Biology Society (EMBC), Aug. (2014).

[10] Xinchao Song, Yishang Geng, Distributed community detection optimization algorithm for complex networks, Journal of Networks, 9(10), 2758-2765, Jan. (2014).

[11] Jiang D, Ying X, Han Y. Collaborative multi-hop routing in cognitive wireless networks[J]. Wireless Personal Communications, (2015): 1-23.

[12] Jinyu Hu and Zhiwei Gao. Modules identification in gene positive networks of hepatocellular carcinoma using Pearson agglomerative method and Pearson cohesion coupling modularity[J]. Journal of Applied Mathenatics, 2012 (2012).

[13] Jiang $\mathrm{D}, \mathrm{Xu} \mathrm{Z}$, Chen $\mathrm{Z}$. Joint time-frequency sparse estimation of large-scale network traffic[J]. Computer Networks, 2011, 55(15): 3533-3547.Jinyu Hu, Zhiwei Gao and Weisen Pan. Multiangle Social Network Recommendation Algorithms and Similarity Network Evaluation[J]. Journal of Applied Mathematics, 2013 (2013).

[14] Mingda Zhou, Guanqun Bao, Yishuang Geng, Bader Alkandari, Xiaoxi Li, Polyp detection and radius measurement in small intestine using video capsule endoscopy, 2014 7th International Conference on Biomedical Engineering and Informatics (BMEI), Oct. (2014).

[15] Gan Yan, Yuxiang Lv, Qiyin Wang, Yishuang Geng, Routing algorithm based on delay rate in wireless cognitive radio network, Journal of Networks, 9(4), 948-955, Jan. (2014).

[16] Lin Y, Yang J, Lv Z. A Self-Assessment Stereo Capture Model Applicable to the Internet of Things[J]. Sensors, (2015), 15(8): 20925-20944.

[17] Wang K, Zhou X, Li T. Optimizing load balancing and data-locality with data-aware scheduling[C]. Big Data (Big Data), 2014 IEEE International Conference on. IEEE, (2014): 119-128. 


\section{Author}

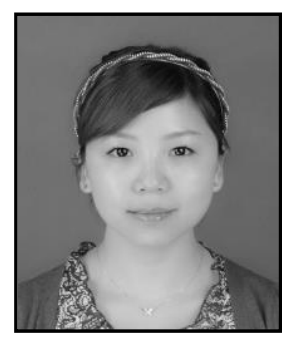

Shan Du, She received her M.S. degree in Artistic Design from Shaanxi University of Science \& Technology in Xi'an, China. She is currently a lecturer in the College of Art and Design at Zhengzhou University of Aeronautics. Her research interest is mainly in the area of Industrial Design, Product Design Theory. She has published several research papers in scholarly journals in the above research areas and has participated in several books.

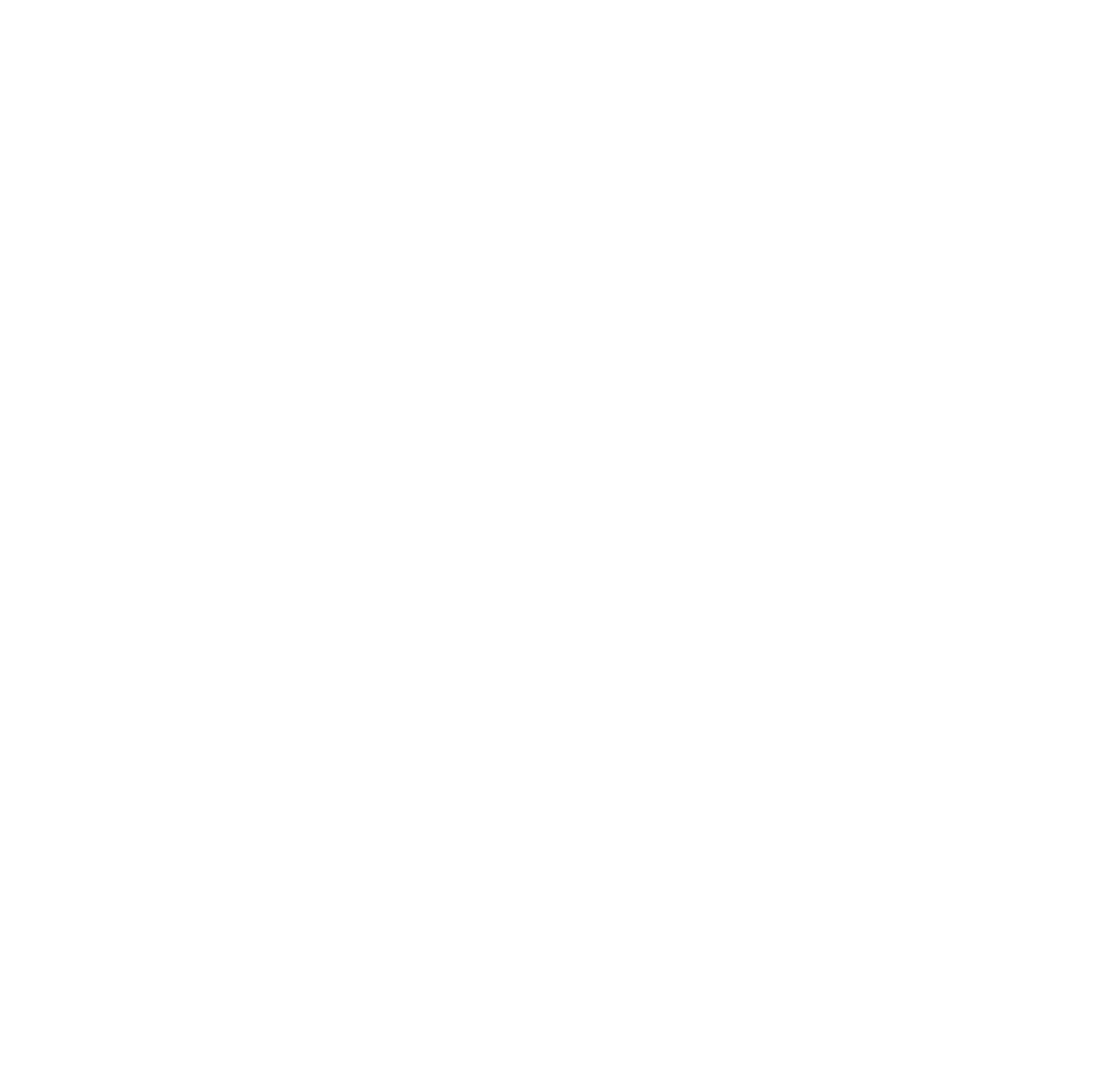


International Journal of Smart Home

Vol.10, No.10 (2016)

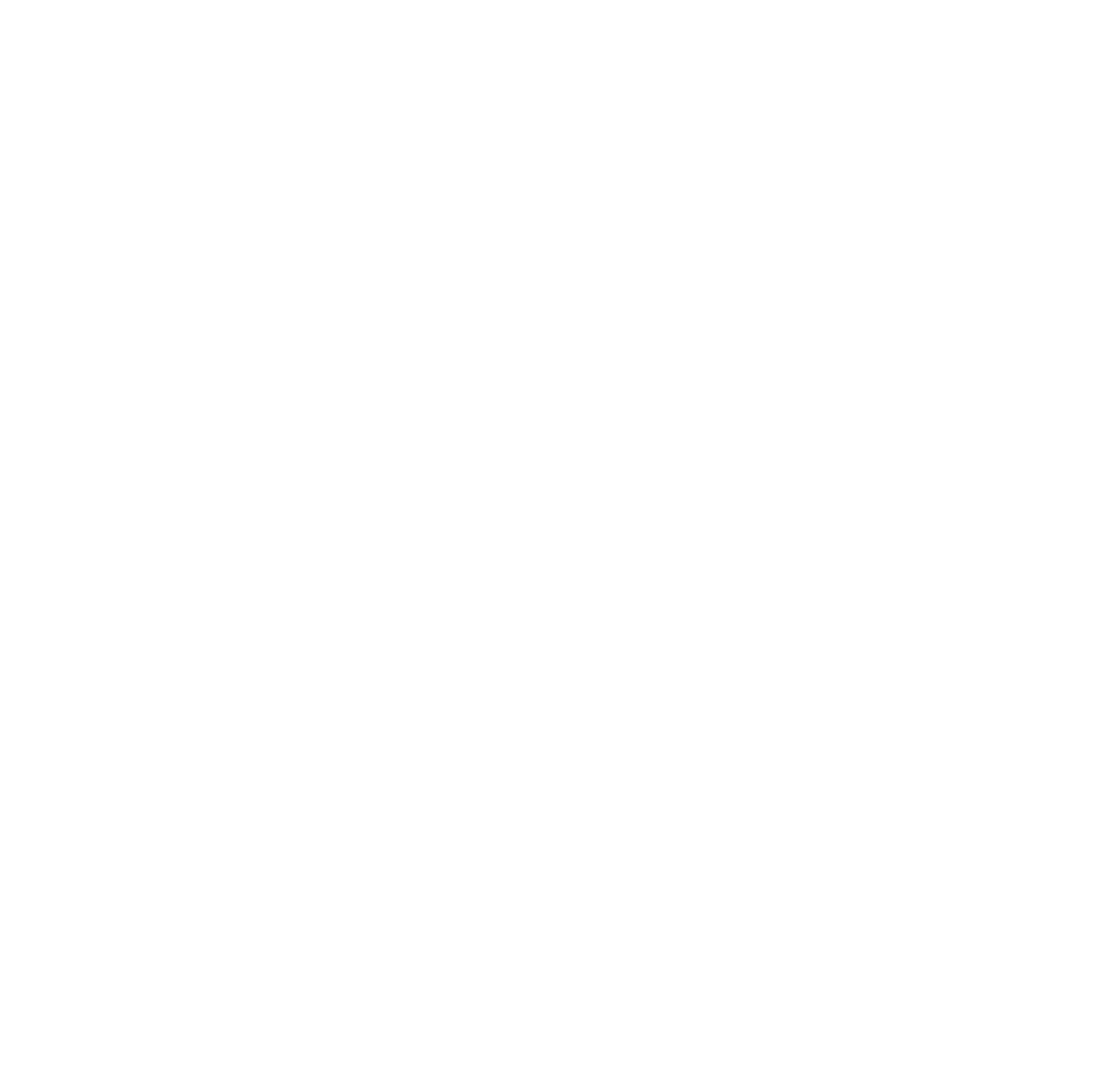

